\title{
EXHIBITION OF
}

\section{AMERICAN HANDICRAFTS}

\author{
ASSEMBLED AND CIRCULATED
}

$\mathrm{BY}$

THE AMERICAN FEDERATION OF ARTS

$1922-23$

NATIONAL GALLERY OF ART

UNITED STATES NATIONAL MUSEUM 



\section{CATALOGUE}

\section{EXHIBITION OF}

\section{AMERICAN HANDICRAFTS}

ASSEMBLED AND CIRCULATED

BY

THE AMERICAN FEDERATION OF ARTS

$1922-23$ 


\section{THE AMERICAN FEDERATION OF ARTS}

\section{SPECIAL COMMITTEE ON HANDICRAFTS*}

H. P. Macomber, Sec'y, Boston Society of Arts and Crafts, Chairman

George G. Воoth, Pres., Detroit Society of Arts and Crafts

F. A. Whiting, Director, Cleveland Museum of Art

Samuel Yellin, Iron Worker of Philadelphia

Charles Pellew, Pres., Nere York Society of Craftsmen

\section{JURY OF SELECTION}

C. Howard Walker, Architect and President of the Boston Society of Arts and Crafts

Huger Elliotr, Director, Pennsylvania Museum School of Industrial Art

Bertram G. Goodhue, Architect, New York

Sarah W. Hendrie, Chairman Jury, Detroit Society of Arts and Crafts

Francis C. Jones, N. A., Chairman, Exhibition Committee of The American Federation of Arts

Henry W. Kent, Secretary, Metropolitan Museum of Art, Nerw York, and President of the Grolier Club

Hermann Rosse, Designer, Instructor Chicago Art Institute

*Members ex officio Jury of Selection 


\section{FOREW ORD}

This exhibition of American Handicrafts circulated under the auspices of the American Federation of Arts, offers unmistakable evidence that, in the twenty-five years since the "first exhibition of the Arts and Crafts" held in Boston in 1897, there has been very encouraging progress both in craftsmanship and design. During this period the opportunity for the ready sale of good handicraft has been demonstrated and this work has undoubtedly played a part in raising the standard of similar work manufactured commercially. The Committee believes that this exhibition affords a creditable representation of the present stage of our craftsmen in designing and executing objects which are both useful and beautiful. No claim is made that it comprises all the good work now being done, for the time allowed for its preparation and collection was rather short. We hope that the work shown will introduce American handicrafts to a wider public and serve as an inspiration and encouragement for many others to enter this field. In the further development of handicraft, it is the abuse and not the use of the machine which is to be condemned, and it is the importance of good design which cannot be too strongly insisted on.

Grateful acknowledgment is made of the cooperation of the Smithsonian Institution, the National Museum, and the National Gallery of Art in assembling this exhibition and giving it first showing; and most particularly of the active and invaluable expert assistance of Mr. William H. Holmes, Director of the National Gallery of Art, and his staff.

H. P. Macomber, Chairman,

Committee on Handicrafts A. F. A. 


\section{MUSEUMS PARTICIPATING IN THIS EXHIBITION}

National Gallery of Art, Washington, D. C.

Pennsyluania Museum, Philadelphia, Pa.

Museum Rhode Istand School of Design, Providence, $R$. $I$.

Museum of Fine Arts, Boston, Mass.

Carnegie Institute, Pittsburgh, $P a$.

Museum of Art, Cleveland, Ohio.

\section{Notice}

Almost all the articles in the exhibition are for sale; prices may be had on application.

No article can be delivered to the purchaser until the close of the circuit, as it is necessary to preserve the integrity of the exhibition until the end.

When articles are lent or not for sale, replicas can be ordered in many cases. These can be delivered to the purchaser as soon as received. 


\section{CATALOGUE}

\section{JEWELRY}

1. Pendant; gold and emeralds. Amy V. Beal, Brookline, Mass.

2. Pendant; carved gold cross. John Sydney Burton, Cleveland, Ohio.

3. Locket; carved gold with "Little Boy" in carved ivory. John Sydney Burton, Cleveland, Ohio.

4. Pendant; silver and enamel with carved ivory. JohN Sydney Burton, Cleveland, Ohio.

5. RING; large green tourmaline set in platinum with emeralds and diamonds. Lucretia McMurtrie Bush, Boston, Mass.

6. Pendant and Chain; gold with black opals, diamonds, saphires and pearls. Jessie Ames Dunbar, Boston, Mass.

7. Pendant; silver, enamel and moonstones. Mrllicent Strange Edson, Lakemont, Georgia.

8. Pendant; gold, enamel emeralds, peridots and moonstones. Millicent Strange Edson, Lakemont, Georgia.

9. RING; gold, pink ruby with small diamonds. MARY A. Frye, Boston, Mass.

10. Pendant; gold, berry design, carved ivory. Tracy $A$. Griffing, Eastport, N. Y.

11. Pendant; gold, white and green jade, lapis and enamel. Frank Gardner Hale, Boston, Mass. (Owned by Mrs. Oliver Hershman.)

12. Necklace; Mexican fire opal. Grace Hazen, New York City.

13. Necklace; gold, pink tourmaline and gold beads. Emma G. Hunt, Boston, Mass.

14. Ring; silver, gold and moss agate. Malcolm E. Johnson, Boston, Mass.

15. Cuff Links; gold and jade. Herbert Kelley, Croton-onHudson, N. Y.

16. Necklace; gold and tourmalines. Herbert Kelley, Croton-on-Hudson, N. Y.

17. Pendant; gold, opals and olivines. Herbert Kelley, Creton-on-Hudson, N. Y.

18. Brooch; precious topaz, diamonds and pearls. Edward E. OAKEs, Boston, Mass. 
19. Cross; gold, sapphires and pearls. Reginald F. Pearce, Boston, Mass.

20. Brooch; gold, yellow sapphire. Gertrude Peet, Cambridge, Mass.

21. Ring; gold, enamel and black opal. The Potter Studio. (Designed by Florence L. Potter; executed by C. H. Kurz, J. S. Burton and H. E. Potter.) Cleveland, Ohio.

22. Brooch; gold, enamel, green zircon and pearls. The Ротter Studio, Cleveland, Ohio. (Designed by H. E. Potter; executed by. J. S. Burton and H. E. Potter.)

23. Pendant and Chain; gold, enamel and Mexican opals. The Potter Studio, Cleveland, Ohio. (Designed by $\mathrm{H}$. E. Potter; executed by C. H. Kurz, J. S. Burton and H. E. Potter.)

24. Cross; moonstone and black onyx, gold and silver. MARgaret Rogers, Boston, Mass.

25. Ring; opal, diamond and olivine. Magaret Rogers, Boston, Mass.

26. Chain; silver and black onyx. Grace Schriefer, Burlingame, Calif.

27. Ring; silver and black onyx. Grace Schriefer, Burlingame, Calif.

28. Brooch; gold, pearls and star sapphire. HeLen Sweetser White, Philadelphia, $\mathrm{Pa}$.

29. Bracelet; gold and aquamarines. Helen Sweetser White, Philadelphia, Pa.

30. Pendant and Chain; gold, moonstone and garnets. Helen Sweetser White, Philadelphia, Pa.

31. Necklace; gold, pearls and black opals. Helen Sweetser White, Philadelphia, Pa.

32. Ring; gold and pink tourmalines. Helen Sweetser White, Philadelphia, Pa.

\section{ENAMELS}

33. Vanity Box; carved ivory, silver, cloisonne enamel. EdA Lord Dixon, Riverside, Calif.

34. Chalice; silver and enamel. Eda Lord Dixon, Riverside, Calif.

\section{CARVED IVORY}

35. The "B" Collection of Medallions. Nathan S. Brody, Cleveland, Ohio. 


\section{SILVER}

36. Bowl; silver. Porter Blanchard, Gardner, Mass. (Designed by Porter Blanchard; executed by David Carlson.)

37. Chalice; silver and amethyst. Porter Blanchard, Gardner, Mass. (Executed by David Carlson.)

38. Six-Piece Service; silver. George G. Gebelein, Boston, Mass.

39. Bowl; fluted silver. F. J. R. Gyllenberg, Boston, Mass. 40. Covered Bowl; silver. Elizabeth Hadjisky, Detroit, Mich.

41. Bowl; silver. Karl F. Leinonen, Boston, Mass.

42. Bowl; silver. Helen K. Mirls, Kent, Conn.

43. "The President Eliot Cup"; silver. Arthur J. Stone, Gardner, Mass. Adapted by Prof. H. Langford Warren from the Greek. (Executed by A. J. Stone and William Blair.)

44. Teapot.

45. Sugar and Cream.

Arthur J. Stone, Gardner, Mass. (Executed by A. J. Stone and assistants: H. W. Taylor, A. L. Hartwell, E. H. Renderwood, P. H. Harrison.)

46. Chalice; silver. Arthur J. Stone, Gardner, Mass. (Executed by A. J. Stone and his assistants.)

47. Salt Spoon; fiddlehead. Arthur J. Stone, Gardner, Mass.

48. Cream Ladle; fiddlehead. Arthur J. Stone, Gardner, Mass. (Executed by Charles W. Brown.)

49. Punch Ladie. Arthur J. Stone, Gardner, Mass. (Executed by A. J. Stone and Charles W. Brown.)

50. Serving Spoon. Arthur J. Stone, Gardner, Mass. (Designed by A. J. Stone; executed by Charles W. Brown.)

51. Round Fluted Bowl. James T. Woolley, Boston, Mass.

52. Round Fluted Bowl. James T. Woolley, Boston, Mass.

53. Chocolate Pot and Tray. James T. Woolley, Boston, Mass.

54. Fluted Teapot and Tray. James T. Wooley, Boston, Mass.

55. Porringer. James T. Woolley, Boston, Mass.

PEWTER

56. Fruit Bowl. Louis F. Grandmont, Taunton, Mass.

57. Pitcher. Lester H. Vaughan, Taunton, Mass.

58. Bowl. Lester H. Vaughan, Taunton, Mass.

59. Porringer. Lester H. Vaughan, Taunton, Mass. 


\section{IRON}

60. Stair-Rail Panel. F. Krasser \& Co., Roxbury, Mass.

(Designed by Coolidge \& Shattuck, architects; executed by Krasser \& Co.; lent by R. T. Crane, Jr.)

61. Trivet with Brass Top. Middlebrook Forge, Dover,

N. H. (Designed and executed by Roscoe E. Grant.)

62. Tonsting Fork. Middhebrook Forge, Dover, N. H. (Designed and executed by Roscoe E. Grant.)

63. Pipe Tongs. Middlebrook Forge, Dover, N. H. (Executed by Jacob Rinta.)

64. Hearth Fork. Middlebrook Forge, Dover, N. H. (Designed and executed by Roscoe E. Grant.)

65. Gothic Chest and Stand. Samuel Yeluin, Philadelphia.

\section{POTTERY}

66. Aubergine Bown, lined turquoise. Arden Studios, Inc., New York, N. Y. (Designed and executed by Leon Volkmar, Durant Kilns.)

67. Apple-green Bowl. Arden Studios, Inc., New York, N. Y. (Designed and executed by Leon Volkmar, Durant Kilns.)

68. Ox-blood Vase. Arden Studios, Inc., New York, N. Y. (Designed and executed by Leon Volkmar, Durant Kilns.)

69. Celladon and Willow-gray Vase. Prof. Charles F. Binns, Alfred, N. Y.

70. Vapor Blue Vase. Prof. Charles F. Binns, Alfred, N. Y.

71. Figurine; mediaeval, orange. Dora Clarke, Detroit, Mich.

72. Figurine; mediaeval, yellow. Dora Clarke, Detroit, Mich.

73. Orange Bowl. Ede De F. Curtis, Conestoga Pottery, Wayne, $\mathrm{Pa}$.

74. Bottle Vase; cucumber green. Fulper Pottery, Flemington, N. J. (Designed by W. H. Fulper.)

75. Low-handled Jar. Fulper Pottery Co., Flemington, N. J. (Designed by W. H. Fulper.)

76. Low Ovoid Vase, with Neck. Fulper Pottery, Flemington, N. J. (Designed by W. H. Fulper.)

77. Persian Plate. George C. Greener, Boston, Mass.

78. Persian Plate. George C. Greener, Boston, Mass.

79. White Bowl and Stand. Sarah H. Groom, Brookline, Mass. 
80. Vase and Base. Greenwich House Pottery, New York,

N. Y. (Designed by Martha de Michel.)

81. Bowl. Jessie F. Gordon, Philadelphia, Pa.

82. Covered Dish. Jessie F. Gordon, Philadelphia, Pa.

83. Leopard-skin Bowl and Stand; majolica. Mrs. George H. Hobson, Brookline, Mass.

84. Long-necked Persian Bottle; semi-faience 1700-1750.

Mrs. George H. Hobson, Brookline, Mass. 、

85. Rakka Mesopotamian Vase; XI and XII centuries. Mrs. George H. Hobson, Brookline, Mass.

86. Bowl; turquoise and black. The Marblehead Potteries, Marblehead, Mass. (Designed by Arthur E. Baggs; lent by Frank G. Macomber.)

87. Vase; turquoise and black. The Marblehead Potteries, Marblehead, Mass. (Designed by Arthur E. Baggs.)

88. Vase; wisteria and violet. The Marblehead Potteries,

Marblehead, Mass. (Designed by Arthur E. Baggs.)

89. Vase; gray, green and tan. The Marblehead Potteries,

Marblehead, Mass. (Designed by Arthur E. Baggs.)

90. Jar with Cover; turquoise. Gertrude Burgess, N. Y.

State School of Ceramics, Alfred, N. Y.

91. Jar with Cover; orange. Audrey Haynes, N. Y. State School of Ceramics, Alfred, N. Y.

92. Jar with High Cover; red orange. Mildred Andrews,

N. Y. State School of Ceramics, Alfred, N. Y.

93. Decorated Bowl. Newcomb Pottery, New Orleans, La.

(Designed by S. E. Irvine.)

94. Decorated Vase. Newcomb Pottery, New Orleans, La. (Designed by Corinne Chalaron.)

95. Bowl. Paul Revere Pottery, Brighton, Mass.

96. Bowl and Flower-Holder. Paul Revere Pottery, Brighton, Mass. (Designed by Edith Brown; executed by Eva Genecco.)

97. Vase. Paul Revere Pottery, Brighton, Mass. (Designed by Edith Brown; executed by Lillian Shapiro.)

98. Vase; new Dixon ivory. Paul Revere Potrery, Brighton, Mass. (Designed by Edith Brown; executed by Miss Lipson.)

99. Six Miniature Vases; porcelain. Adelaide Alsop RobiNEAU, Syracuse, N. Y.

a. "Swarm of Gnats," crystals.

b. Turquoise opalescent drip.

c. Light orange crystals. 
d. Blue crystals, pear-shaped.

e. Rare red crystals.

f. Lavender-on-buff crystals.

100. Covered Jar; porcelain, "Bee Swarm." Adelaide Alsop Robineau, Syracuse, N. Y.

101. Vase; white crystals, porcelain. Adelaide Alsop Robineau, Syracuse, N. Y.

102. VASE; rare crystalization, porcelain. AdelaIde Alsop Robineau, Syracuse, N. Y.

103. Carved Bowl; "Peruvian Serpent," porcelain. Adelaide Alsop Robineau, Syracuse, N. Y.

104. Glazed Pottery Relief in Frame, "Mother and Child."

Alice B. Stone, Jamaica Plain, Mass.

105. White Bowl and Stand. Alice M. Sturgis, Boston, Mass.

106. Pitcher. Paul St. Gaudens, Windsor, Vermont.

107. Blue Jar. Paul St. Gaudens, Windsor, Vermont. (Lent by Naomi Rhodes.)

108. VASe with Cover and Stand; green crackle-glaze. Irene

E. Burtt, Wylie, N. Y.

109. Vase; plain. The Rookwood Pottery Co., Cincinnati, O. (Designed by J. D. Wareham.) "Tiger-eye."

110. Conventional Bowl; decorated enamel. The Rookwood

Pottery Co., Cincinnati, O. (Designed by K. Shirayama; decorated by W. E. Hentschel.)

111. Vase; plain, "Rouge Flambé." The Rookwood Pottery Co., Cincinnati, O. (Designed by J. D. Wareham.)

112. VAse; plain, "Rouge Flambé," deep. The Rookwood PoxTERY Co., Cincinnati, O. (Designed by J. D. Wareham.)

113. Box and Cover; mottled blue, plain soft porcelain. The Rookwood Potrery Co., Cincinnati, O. (Designed by J. D. Wareham.)

114. Faience Tile. The Rookwood Pottery Co., Cincinnati, O. (Designed by W. P. McDonald.)

\section{DECORATED CHINA}

115. Pitcher. Nina Hatfield, Hoboken, N. J.

116. Plate; XIII Century Persian. Grace H. Hackett, West Roxbury, Mass.

117. Satsuma Vase; Coptic design. Mildred C. Kingsbury, Baltimore, Md.

118. Plate; enamel. Henrietta M. King, Detroit, Mich. 
119. Bowl; enamel. Henrietta M. King, Detroit, Mich.

120. Satsuma Bowl; enamel. Henrietta M. King, Detroit, Mich.

121. Satsuma Box; hexagonal. L. Althea Mowbray, Minneapolis, Minn.

\section{TEXTILES}

\section{Batik and Block-Printed Textiles}

122. Blue Hanging. Maud Arnslie, Louisville, Ky.

123. Wall Hanging, "Ulysses." Theodore Bolton, Paterson,

N. J. (Executed by Helen Stevens.)

124. Panel, Silk, "Blue Waterfall." Lydia Bush-Brown, New York, N. Y.

125. Wall Hanging, Silk, "The Tropics." Lydia BushBrown, New York, N. Y.

126. Blocked Table Square. Helma Boeker, Richmond Hill, N. Y.

127. Table Scarf. Mary Susan Collins, Cleveland, Ohio.

128. Wall Hanging. Mary Susan Collins, Cleveland, Ohio.

129. Wall Hanging, "San Francisco." E. Shotwell Goeller, San Francisco, Calif.

130. Blocked and Stitched Bag. Jennie Hirsch, Rutherford, N. J.

131. Negligee. Jennie Hirsch, Rutherford, N. J.

132. Hanging, "A Song to the Morning Star." L. Althea Mowbray, Minneapolis, Minn.

133. Wall Hanging, "Brown Country in Autumn." Charles B. Mrllholland, Indianapolis, Ind.

134. Panel, "Pierrot's Dream." Adelaide Baker (Morgan), Washington, D. C.

135. Hanging, "Ivan, Illia and the Palace." Marion Crane Maerchlein, Hartford, Conn.

136. Hanging, "The Elephant." Marion Crane Maercklein, Hartford, Conn.

137. Hanging, "The Golden Buddha." Marion Crane Maercklein, Hartford, Conn.

138. Hanging, "Lion and Lambs." Marion Crane Maercklein, Hartford, Conn.

139. Hanging, "Flower Print in Twenty-two Blocks." Marion Crane Maercklein, Hartford, Conn.

140. Hanging, "The Dancing Grrls." Marion Crane Maercklein, Hartford, Conn. 
141. Hanging, "The Arbor." Marion Crane Maercklein, Hartford, Conn.

142. Decorated Scarf. Noank Studio, New York, N. Y.

143. Decorated Scarf. Noank Studio, New York, N. Y.

144. Hanging. Helen Stevens, Washington, D. C.

145. Scarf. Elizabeth W. Shannon, Baltimore, Md.

146. Block Printed Table Cover. Elizabeth W. Shannon, Baltimore, Md.

147. Over-mantel, "In the Park." Jean Paul Slusser, New York, N. Y.

148. Hanging, "Fruit, Birds and Flowers." Jean Paul Slusser, New York, N. Y.

149. Hanging, "Tree of Life." Jean Paul Slusser, New York, N. Y.

150. Batik, "Jar with Flowers." Nell Witters, Chicago, Ill. 151. Batik, "The Castle Garden." Nell Witters, Chicago, III.

152. Batik, "Her Sampler." Nell Witters, Chicago, III.

\section{Weavings}

153. Linen Towel. Flambeau Shops, Inc., New York, N. Y.

154. Handwoven Runner. Flambeau Shops, Inc., New York, N. Y.

155. Curtain; chain mail, grenadine weave. Flambeau Shops, INc., New York, N. Y.

156. Silk Cover; handwoven. Flambeau Shops, Inc., New York, N. Y.

157. Woven Square. Mary P. Hibbard, New York, N. Y. 158. Table Runner. Mrs. E. C. Richards, Highland Park, Detroit, Mich.

159. Linen Luncheon Set. Mrs. E. C. Richards, Highland Park, Detroit, Mich.

160. Woven Woolen Scarf. The Tenafly Weavers, Tenafly, N. J.

161. Woven Runner. The Tenafly Weavers, Tenafly, N. J. 162. Woven Runner. The Tenafly Weavers, Tenafly, N. J. 163. Small Woven Runner. The Tenafly Weavers, Tenafly, N. J.

164. Design in Silk. Anna H. Taylor, Columbia, S. C.

165. Woven Coverlet, "Whig Rose" Design. Nina B. Forsythe, Berkeley, Calif.

166. Woven Coverlet, "De Fronsac" Design. Nina B. Forsythe, Berkeley, Calif. 
167. Woven Coverlet, "Whig Rose" Variation. Nina B. Forsythe, Berkeley, Calif.

168. Woven Runner, "Roses." Nina B. Forsythe, Berkeley, Calif.

\section{Needlerwork}

169. Small Scarf; embroidered blue silk. Mabel P. Cook, Lexington, Mass.

170. Needlepoint Panel. Olive Earle, New York, N. Y. (Designed by J. P. Slusser.)

171. Runner. Folk Handicrafts Guild, Boston, Mass. (Designed by Elwyn G. Gowen; executed by Mrs. Andreole.) 172. Table Cover. Folk Handicrafts Guild, Boston, Mass.

(Designed by Elwyn G. Gowen; executed by Mrs. DelCare.)

173. Table Cover. Folk Handicrafts Guild, Boston, Mass.

(Designed by Elwyn G. Gowen; executed by Concentta Bionals.)

174. Knot Tapestry (inset in silver box). Iza A. Grigsby (box by Karl F. Leinonen), Columbus, Ohio.

175. Woollen Table Scarf; Jacobean embroidery. Guild of Thread and Needle Workers of the Society of Arts and Crafts, Boston, Mass. (Designed by Grace Stratton.)

176. Peacock Gobelin Brooch, Petit Point. Mary Galdonyi, Detroit, Mich.

177. Italian Linen Square. Mildred Mowll, Cambridge, Mass.

178. Lace Collar. Teresa Pellegrini, Boston, Mass.

179. Panel; angel embroidered on velvet. Sister Olive Frances, Convent of St. John Baptist, Ralston, N. J.

180. White Chalice Veil; embroidered on linen. Sister Olive Frances, Convent of St. John Baptist, Ralston, N. J.

181. Embroidered Saint Paul (Panel). Sister Olive Frances, Convent of St. John Baptist, Ralston, N. J.

\section{BOOKBINDING}

182. Altar Service Book. Eliza Ingle, Baltimore, Md.

183. Altar Service Book. The Misses Lloyd, Detroit, Mich. 184. "The Cock and Hen," by Chaucer. John F. Grabau, Buffalo, N. Y. 
185. Magazine Cover. Miriam B. Pearce, Hillside Studio, Newton, Mass.

186. Seven Poems, by Tennyson. Mary Crease Sears, Boston, Mass.

187. Areopagitica. Mary Crease Sears, Boston, Mass.

\section{ILLUMINATIONS}

188. Prayers, by R. L. Stevenson. Gertrude S. Bassett, Boston, Mass.

189. Prayer, framed. Wilhelmina R. Gillooly, Wakefield, Mass.

190. Psalm of Good Teacher, on vellum. Sister Olive Frances, Convent of St. John Baptist, Ralston, N. J.

191. Consecration Prayer, on vellum (unfinished). Sister Olive Frances, Convent of St. John Baptist, Ralston, N. J.

192. Drawing, on vellum. George P. West, Philadelphia, Pa.

\section{BOOK PLATES}

193. Frame with Book Plates. Alexandrine McEwen, Detroit, Mich.

194. Wood Block Print on Paper. Nan R. Miller, Boston, Mass.

\section{DESIGNS FOR ADVERTISEMENT}

195. Design. Walter Darwin Teague, New York, N. Y. 196. Design. Walter Darwin Teague, New York, N. Y.

197. Design for Catalogue Covers. Walter Darwin Teague, New York, N. Y.

198. Design for Catalogue Covers. Walter Darwin Teague, New York, N. Y.

\section{STAINED GLASS}

199. "Sr. Augustine," replica of panel in St. John's Church, Beverly Farms, Mass. Charles J. Connick, Boston, Mass.

200. "Service," replica from Chapel of Boston University. Charles J. Connick, Boston, Mass.

201. Medallion, English painted glass. Margaret Redmond, Chesham, N. H. 
202. Panel, "The Crucifixion." Reynolds, Francis \& Rohnsтоск, Boston, Mass. (Designed by Joseph G. Reynolds, Jr.; executed by William M. Francis and J. Henry Rohnstock.)

\section{WOOD CARVING}

203. Frame. Carrig Rohane Shop, Inc., Boston, Mass. (Designed by H. D. Murphy.)

204. Miniature Frame. John E. Eckberg, Boston, Mass. (Designed by Adrian Eckberg.)

205. Mahogany Carving of Man, Title "Peter." Robert $J$. Kunn, Richmond Hill, L. I.

206. "Madonna and Chind." John Kirchmayer, Cambridge, Mass.

207. Box; green and gold. Walfred Thulin, Boston, Mass. 208. Eagle Mrrror; carved gold and silver. Walfred Thulin, Boston, Mass.

209. Mrrror; carved blue and gold. Walfred Thulin, Boston, Mass.

210. Ship Model. Henry B. Culver, New York, N. Y.

\section{LACQUER}

211. Covered Bowl. Floy K. Hanson, New York, N. Y. 212. Lacquer Panels for Cabinet Doors. Floy K. Hanson, New York, N. Y. 


\section{THE AMERICAN FEDERATION OF ARTS}

The American Federation of Arts was formed in 1909 to 1 carry on "the eternal propaganda of beauty," by spreading the knowledge and appreciation of Art.

It assembles and sends out traveling exhibitions of paintings, prints, design, handicrafts, etc.;

Circulates illustrated lectures on art;

Publishes The American Art Annual, a directory of art and artists in America, and The American Magazine of Art, an illustrated monthly;

Serves as a Bureau of Information and a general clearing house for art;

Holds an Annual Convention.

All of the leading Art Museums and Associations are Chapters. It has branch offices in New York City and Lincoln, Nebraska.

Individual members are classified as follows:

Associate members ................ \$5.00 a year

Active " $\quad \ldots \ldots \ldots \ldots \ldots \ldots \ldots \ldots \ldots, 10.00$ " "

Sustaining " $\ldots \ldots \ldots \ldots \ldots \ldots \ldots \ldots \ldots 100.00$ " "

Life members-one payment .......... 500.00

Perpetual members-one payment .........1,000.00

(Can be bequeathed.)

Main Office:

174t New York Avenue, Washington, D. C. 


\title{
THE AMERICAN FEDERATION OF ARTS
}

\author{
OFFICERS \\ ROBERT W. DE FORSET \\ President \\ CHARLES L. HUTCHINSON \\ First Vice-President
}

\author{
LEILA MECHLIN \\ Secretary \\ HELEN H. CAMBELL \\ Assistant Secretary \\ LAURA JOY HAWLEY \\ Field Secretary
}

CHARLES D. NORTON
Treasurer

IRENE M. RICHARDS

Assistant Treasurer

RICHARD F. BACH

Extension Secretary

\section{VICE-PRESIDENTS:}

Cecilia Beaux, Nerw York

W. K. Bixby, St. Louis

E. H. Blashfield, Nerw York

Mrs. George Blumenthal, Nerw York

GlenN Brown, Washington

C. T. Crocker, San Francisco

F. A. Delano, Washington

A. E. Gallatin, Newu York

William O. Goodman, Chicago

Morris Gray, Boston

A. A. Hammerschlag, Pittsburgh

Edgar L. Hewett, Santa Fe
Archer M. Huntington, Nerw York RALPH KING, Cleveland

Alexander R. Lawton, Savannah

John F. Lewis, Philadelphia

E. D. LiBbey, Toledo

A. W. Mellon, Washington

Thomas Nelson Page, Washington John Barton Payne, Washington William B. Sanders, Cleveland John R. VAN Derurp, Minneapolis Charles D. WalcotT, Washington HeNry White, Washington

$$
\text { BOARD OF DIRECTORS: }
$$

To Serve to 1923

Herbert Adams George G. Booth Charles A. Coolidge Robert W. DE Forest Otto H. KaHN

Charles Allen Munn Mrs. Gustav Radeke G. D. Seymour

To Serve to 1924

Mrs. John W. Alexander John W. Beatty Robert IVoods Bliss Andrew Wright Crawford Charles L. Hutchinson

H. W. Kent Florence N. Levy Elihu Root

To Serve to 1925

Helen C. Frick Cass Gilbert Francis C. Jones R. P. Lamont Charles Moore Charles D. Norton 
